\title{
A dynamic energy-saving strategy for green cellular railway communication network
}

\author{
Jiying Huang ${ }^{1 *}$, Zhangdui Zhong $^{2}$ and Hongwei Huo ${ }^{3}$
}

\begin{abstract}
Energy saving is seldom contemplated in cellular railway communication network because safety is always the main focus. However, we analyzed train schedules and determined that the cellular railway network had considerable potential for energy saving. We designed a simple train detection strategy using the handover procedure to initiate power saving. We also proposed a flexible and effective solution based on the mealy-type finite state machine to transit the cell state between an energy-saving mode and a full running mode. The key design principle of the proposed solution consists in forming an orthogonal group and cluster, based on network deployment and train schedules, to simplify the state transition. Our evaluations using a real network deployment led to a significant energy saving of up to $33 \%$ with different power-saving strategies and factors.
\end{abstract}

Keywords: Green cellular railway network; Energy saving; Finite state machine

\section{Introduction}

Mobile communication networks have experienced tremendous increases over the last 5 years in subscriber/ device quantities, service variety, and traffic loads. This tendency leads directly to ever-increasing energy consumption and $\mathrm{CO}_{2}$ emissions in mobile cellular networks. According to the Green radio report [1], $57 \%$ of the energy consumption of the information and communication technology (ICT) industry is attributed to users and network devices in mobile and wireless networks, the scale of which is still growing exponentially [2]. A quantitative analysis [3] has estimated that ICT contributed to about $2 \%$ of global $\mathrm{CO}_{2}$ emissions in 2007 , and the proportion for cellular networks will double by 2020. Therefore, compared with the traditional design of mobile wireless networks which mainly focuses on ubiquitous access and large capacity, the green cellular wireless network [4-6] has recently received the significant attention, with the aim of developing more energy-efficient systems to make a contribution to reducing the carbon footprint.

Because base stations (BSs) constitute the most significant component of wireless cellular networks, many

\footnotetext{
* Correspondence: EJYING@126.COM

${ }^{1}$ School of Electronic and Information Engineering, Beijing Jiaotong University, No. 3 Shangyuancun, Beijing 100044, China

Full list of author information is available at the end of the article
}

green communication techniques focus on BSs activities. Recently, these green strategies have received increasingly substantial attention from both industry and academia, and several projects such as EARTH, Green Touch, OPERA-Net, Green Radio, and GSMAMEE [7] have been funded for research, deployment, and evaluation. As shown in Figure 1, a typical BS contains three main components of energy consumption: a DC power system, cooling, and a radio unit with RF conversion and signal processing control. According to a research study [8], 62\% of energy consumption came from the radio unit, $25 \%$ of power was consumed by the cooling system, and $11 \%$ by the DC power system. In the literature $[9,10]$, the radio base station system, which uses $10.3 \mathrm{~kW}$ of electricity to produce only $120 \mathrm{~W}$ of effective energy usage for signal transmitting and processing, has quite a low energy efficiency of $1.2 \%$. Therefore, many green strategies $[11,12]$ proposed to switch off the radio unit during its idle time.

In a railway system, the wireless communication platform is the vital control base that administers the operation of the trains and communications among the railway operational staff. With the rapid increase in high-speed railways, a dedicated communication system, currently GSM for railway (GSM-R) in China plays an increasingly important role. GSM-R is widely used in high-speed railways involving passenger transport, and in heavy haulage railways, to provide a communication 


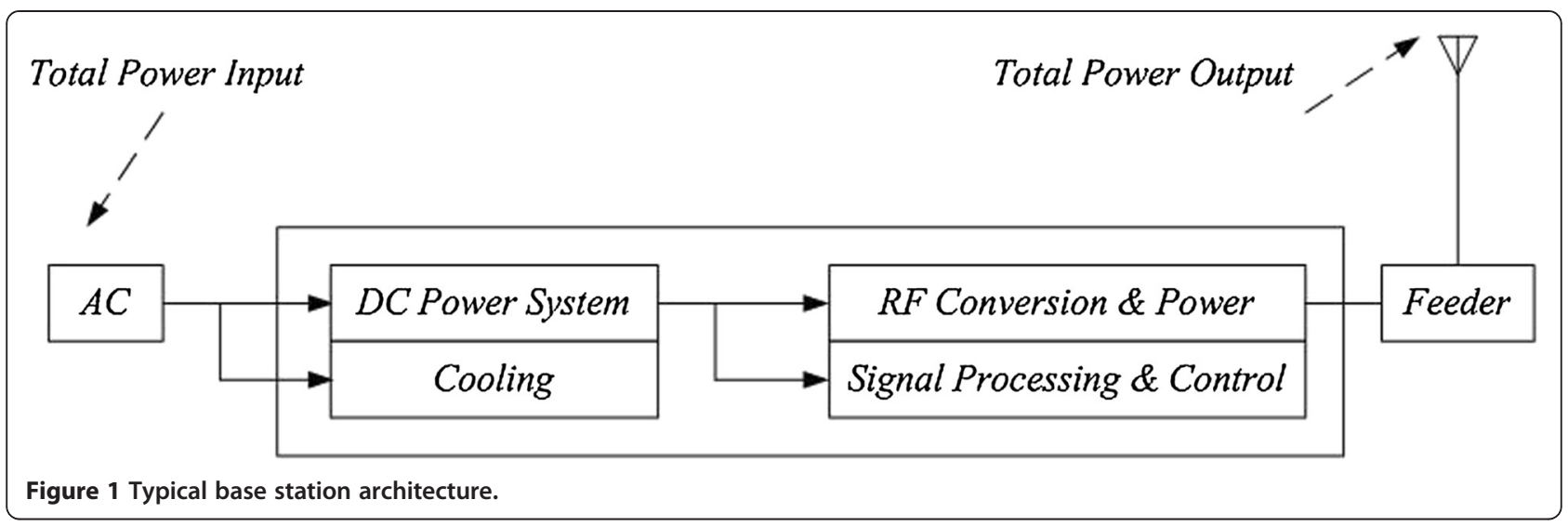

platform for control messages and application services. Twenty-one countries in the world apply GSM-R in railways, such as China, Germany, Italy, and France which have between 3,000 and 4,000 BSs [13]. A single S4/4/4 BS consumes 26,000 kWh per year [14]. Taking $3 \mathrm{~km}$ as an example for the coverage of $1 \mathrm{BS}, 80 \mathrm{BSs}$ in the $240-\mathrm{km}$ railway system consume about 2,080,000 kWh per year. This is an enormous cost and causes large $\mathrm{CO}_{2}$ emissions. From this viewpoint, it is important and urgent to apply power-saving strategies to cellular railway networks.

Compared with a mobile cellular network using a planar coverage scenario, mostly in hot spots, venues, malls, and cities, a dedicated wireless communication network GSM-R deployment has a specific chain structure, and cellular cells are aligned sequentially along railway tracks, as shown in Figure 2. The recent green communication techniques cannot be applied in a dedicated cellular railway network because base stations along the tracks are not allowed to power off due to high security requirements. These security requirements introduce greater challenges for energy saving in a cellular railway network.

In this study, our purpose is to make cellular railway networks more energy efficient. Shown in a real scenario, it is possible to apply the current power-saving strategy to a cell, especially at the idle time; there is less service requirement for railway communication. However, for cellular railway networks, the first question is what event should initiate power saving in a high safety environment. Note that it is impossible to obtain an accurate train schedule in advance. We designed a simple train detection strategy based on the handover process when establishing a communication. Another challenge was to design a mechanism to operate the cell state between an energy-saving mode and a full

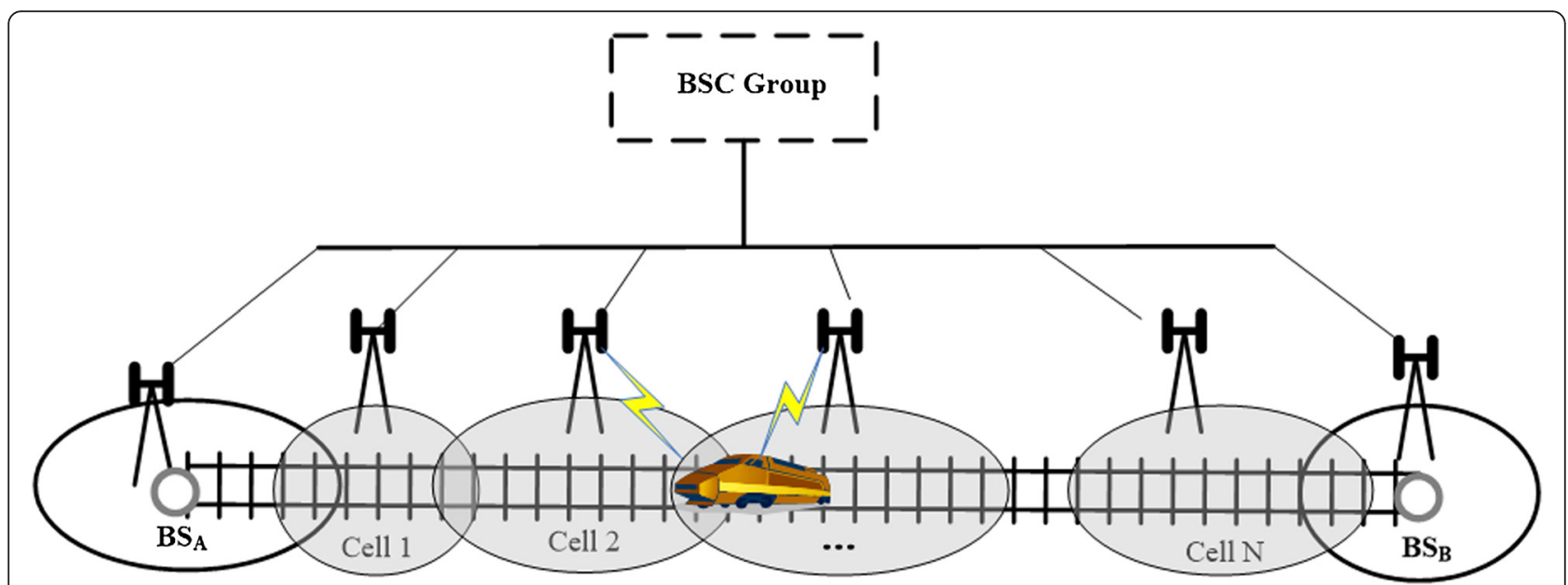

Figure 2 System model for cellular railway networks. 
running mode. Indeed, the trains dynamically run in different cellular regions. We then proposed a flexible and effective solution based on the mealy-type finite state machine. It was shown that its evaluation, using a real network deployment, led to significant energy savings with different power-saving strategies and that the train schedules and speed factors were also verified for our solution. Compared with studies in the literature related to green cellular communication techniques, our contribution covers many aspects:

- To the best of our knowledge, this is the first paper to propose an energy-efficient solution for a green cellular railway network without breaking the rules in the 3GPP Standard.

- We present a new train detection strategy to trigger the power saving which monitors the HANDOVER message in a railway network, rather than obtaining advance the train schedules.

- We propose to take into consideration of the uncertainty of speed measures and bidirectional traffic of trains, and then propose a flexible and effective solution based on a mealy-type finite state machine.

- We provide a detailed algorithm applying a divide and conquer approach in our study, and BSs can easily transfer their state without complicated computation.

- We evaluated our method using the data from real scenarios of railway networks, to identify the many aspects of performance and the impact on the system configuration.

The remainder of this paper is organized as follows. Section 2 discusses related work, and Section 3 provides the model description of the system and proposes the solution and algorithm for two strategies, in detail. Section 4 evaluates the performance using simulation analysis, followed by concluding remarks in Section 5 .

\section{Related work}

Because energy saving and environmental protection have become a global demand and an inevitable trend, the growing awareness of energy efficiency has paved the way for new technologies in designing green cellular networks. Energy efficiency has the advantages of maintaining profitability and reducing the overall impact on the environment. BS has attracted significant attention and has been considered in all stages of cellular networks, especially in the hardware and architecture design, and BS deployment and operation.

Hardware improvements such as using more energyefficient power amplifiers, mean natural resources for cooling, and renewable energy sources can be considered a good approach to achieve energy efficiency. Ericsson
$\Psi$ (Psi) [15] and Flexenclosure E-site solutions [16] are examples of such efforts. A comprehensive survey on how to generate renewable energy is provided in [17], such as solar, wind, and fuel cells, for green base stations in 5G green cellular networks. However, these products and methods are inadequate in dealing with energy waste in a railway cellular network. In railways, it is possible that no trains pass through a specific area for long periods of time, but a cellular operation continues running, resulting in much energy wastage by BSs.

A solution with a new architecture was presented for energy saving, considering current cellular networks cannot meet the design requirements of energy efficiency. The studies $[18,19]$ consider a heterogeneous architecture for network planning. Combining macro-cells with microcells and pico-cells, a theoretical relationship between energy efficiency and network deployment is derived and evaluated. Cognitive radio and cooperative relaying are also significant technologies to improve the throughput and coverage in future networks. A non-stationary spectrum sharing policy to improve the spectrum and energy efficiency is proposed in [20]. These new architectures and technologies cannot be directly used in railway cellular networks because they are complex and lack flexibility. Furthermore, cellular railway networks are not ideal because of the high security requirements.

Because the radio unit, as shown in Figure 1, consumes up to $62 \%$ of the entire energy usage, a dynamically energy-efficient BS operation based on an on/off switching operation can be one of the flexible solutions to reduce the power demands of base stations. Traffic loads in cellular networks have significant features in space and time because of a number of factors such as user mobility and behavior. The BSs are entirely shut down when experiencing low traffic load, and the corresponding traffic loads are transferred to neighboring cells during periods of low use. In [21], the authors suggest the possibility of energy saving using dynamic BS operation based on a simple analytic model from ideal networks. Some cells may be switched off to save energy during daylight for residential areas and at night for office districts. It shows energy savings of the order of 25 to $30 \%$ are possible with several regular cellular topologies. In [22], a profile-based approach was designed to profile base station traffic and approximate the network-wide energy proportionality using non-load-adaptive base stations, and power off of underused base stations under light traffic. This approach is quite suitable for a large, dense city, achieving 53\% energy savings, while a medium-sized city could achieve $23 \%$ energy savings. A similar solution is proposed in [12] to save 50 to $80 \%$ energy in a metropolitan urban area, implementing a distributed approach using a newly introduced notion of network impact to take into account the additional load increments brought to its neighboring BSs. 


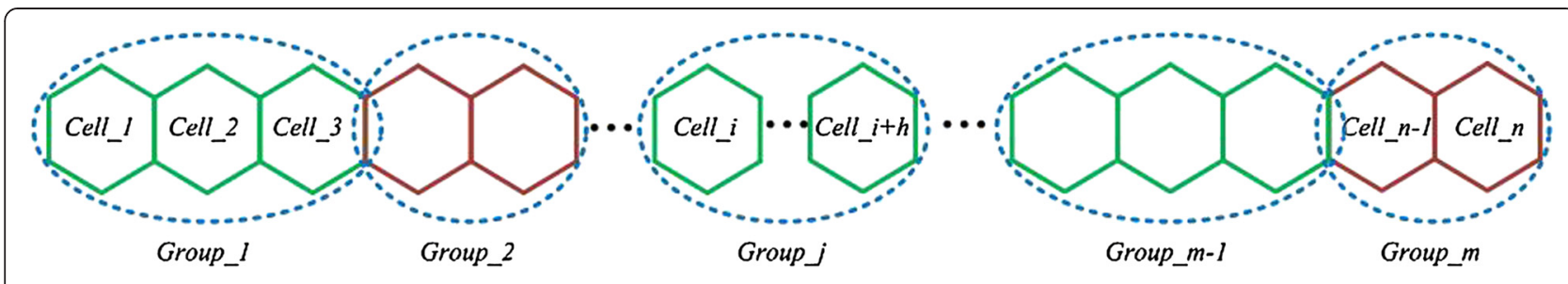

Figure 3 Cell and orthogonal group demonstration.

However, many of these studies only focused on ideal networks, such as a hexagonal model network. They suggested that energy could be saved using dynamic BS switching algorithms. Although other studies have considered actual deployed network scenarios, it was difficult to implement these algorithms in practice because of factors such as complexity, lack of flexibility, and diversity of practical network deployment scenarios. Finally, all these related studies take only regular wireless network deployment scenarios into consideration, instead of focusing on the special wireless network deployment scenarios along railway tracks in which the cell is based on a chain structure instead of a planar structure. In a railway-dedicated mobile network, the traffic load does not follow the daytime/nighttime regular pattern, and the user density is much lower than in public mobile communication networks. Therefore, a new energy efficiency model, based on the internal parameters of a railway cellular network in administering the operation of a network cell, is needed.

\section{System description and the proposed algorithm 3.1 System model}

We consider a wireless cellular railway network which contains a set of $\mathrm{N}$ cells, covering railway tracks from station $\mathrm{BS}_{\mathrm{A}}$ to station $\mathrm{BS}_{\mathrm{B}}$, as shown in Figure 2. The cells in $\mathrm{BS}_{\mathrm{A}}$ and $\mathrm{BS}_{\mathrm{B}}$, which monitor the trains' entry from $\mathrm{BS}_{\mathrm{A}}$ to $\mathrm{BS}_{\mathrm{B}}$, will be active. They only carry out the energy-saving strategy some of the time. Our focus is on dynamic energy saving with active or not strategies for base stations when trains leave the cells controlled by these base stations.

The set of $\mathrm{N}$ cells is controlled by one or several base station controllers (BSCs), and the dynamic energy-saving strategies will be operated by these BSCs.
According to the system model, two main problems arise, for which we will provide corresponding strategies in the following subsections:

(1) How can the cell detect the entry or departure of trains in a particular interval without any prior knowledge of the train schedules?

(2) How can the cell change its energy-saving status given the time-varied distribution of trains on the railway track?

\subsection{Train detection strategy}

It is impossible to obtain the train schedules in advance because of the impact of many factors such as weather, freight requirements, and train status, and it is also not feasible to use the information to schedule the status of the cells. In a GSM-R network, there are two types of special terminals: a vehicle station with higher RF power capability $(8 \mathrm{~W})$ in trains and a normal handheld mobile station (2 W), to transmit and receive messages to control the train schedule.

When a train is scheduled in a typical railway, such as the Beijing-Guangzhou and Zhengzhou-Xian railways in China, a vehicle station will occupy the dedicated wireless channel from the train's start-off until its arrival at its destination. A connection should be initialized between the vehicle station and base station: the vehicle station sends out a Connect Management (CM) SERVICE REQUEST message to the network. The CM SERVICE REQUEST has a RF power-capability element to indicate the type of vehicle station and an International Mobile Subscriber Identity (IMSI). During the traffic channel assignment procedure, one dedicated traffic channel is

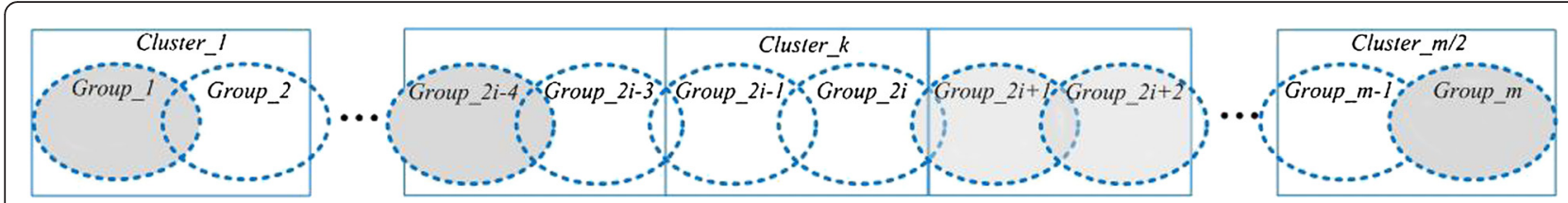

Figure 4 Two orthogonal groups in one cluster. 


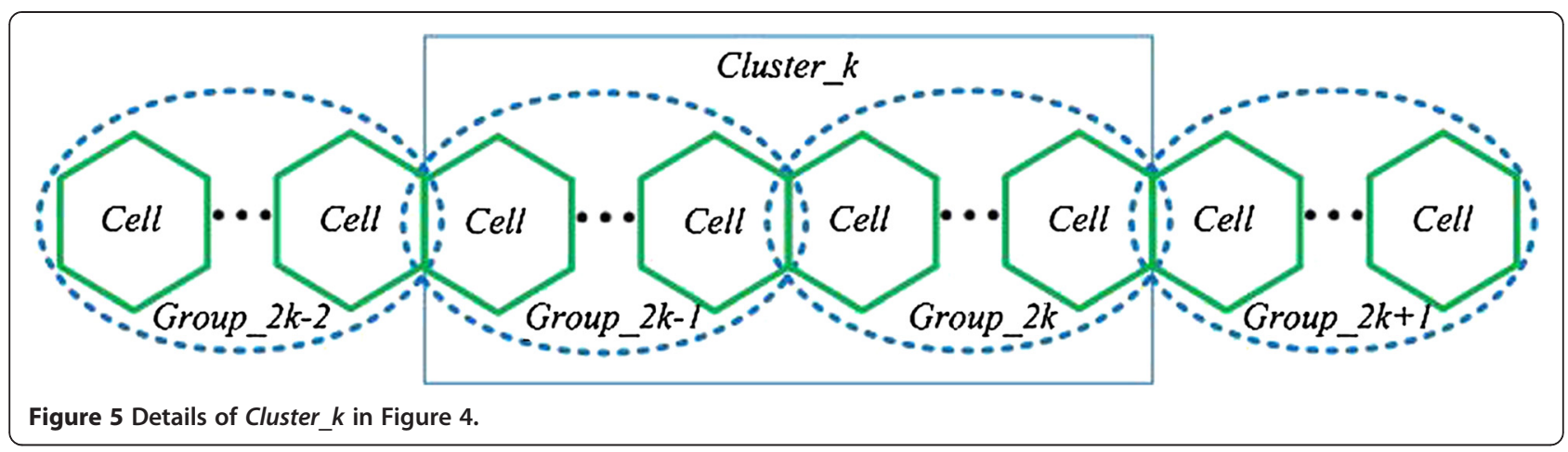

then assigned to the vehicle station, and all the channel properties are contained in the message ASSIGNMENT COMMAND.

When a train enters into the next neighbor cell $i+1$ under base station $i+1$ from cell $i$, the vehicle station on the train $(8 \mathrm{~W})$, as shown in Figure 1, will send out the MEASURE REPORT message to the BSC, which decides whether the vehicle station should start the handover procedure using the message HANDOVER COMMAND. After finishing the successful handover procedure, the vehicle station is now connected to the new cell $i+1$, a HANDOVER COMPLETE message is sent from the vehicle station to the base station $i+1$ to indicate that establishing the new connection is completed. Besides, the old wireless channel in cell $i$ is released by the message RF CHANNEL RELEASE. The message HANDOVER COMPLETE is the indicator that the train has entered the next cell and left the current one.

\subsection{State transition strategy}

The railway characteristics indicate that a cell's status is related to neighboring cells, and the finite state machine model is a powerful method for dealing with cell status transitions. The mealy-type state machine [23], a type of Finite state machine, is based on the present state of the machine, and the input conditions, and can effectively lead to a reduction in the number of states.

\subsubsection{Cell state}

Each cell along the railway tracks consists of two states, namely active and passive. Because there is a high risk to powering off the radio unit in a cellular railway network, the passive state uses a time slot power-saving strategy [24], which was designed to reduce the energy consumption on the idle time slot. The strategy is to adjust the power amplifier bias to a much lower level when no traffic is on the carrier.
When the cell is controlled to be in an energy-saving mode by the BSC node, the corresponding cell enters into the passive state; otherwise, it stays in the active state, which is described as follows:

$$
c_{i}=\left\{\begin{array}{l}
1 \text { (Active), } \mathrm{BS} \text { is not in power saving mode } \\
0 \text { (Passive), BS is in power saving mode }
\end{array}\right.
$$

where $c_{i}$ is the state of cell $i$. The energy-saving mode can be achieved by activating different energy-saving strategies according to the base station's specific requirements.

\subsubsection{Orthogonal group state}

Based on the train detection strategy, first, all the cells along the railway are divided into a number of orthogonal groups without taking into account the overlapped area covered by two adjacent cells. The purpose of forming orthogonal groups is to hide the differences in several special cells that also cover railway stations or residential areas along the railway tracks. On the other hand, some cells have a relatively small radius, and at the same time, the train's speed may be very high, which may lead to insufficient time to perform cell state transition. Using the concept of an orthogonal group, we assume the whole unit performs a state transition. To sum up, the cells are firstly encapsulated to form an orthogonal group to simplify the problem to be solved.

Table 1 States of Cluster_k of Figure 5

\begin{tabular}{lll}
\hline Group_2k-1 & Group_2k & Cluster state \\
\hline 0 (passive) & 0 (passive) & $\mathrm{S}_{00}$ \\
0 (passive) & 1 (active) & $\mathrm{S}_{01}$ \\
1 (active) & 0 (passive) & $\mathrm{S}_{10}$ \\
1 (active) & 1 (active) & $\mathrm{S}_{11}$ \\
\hline
\end{tabular}




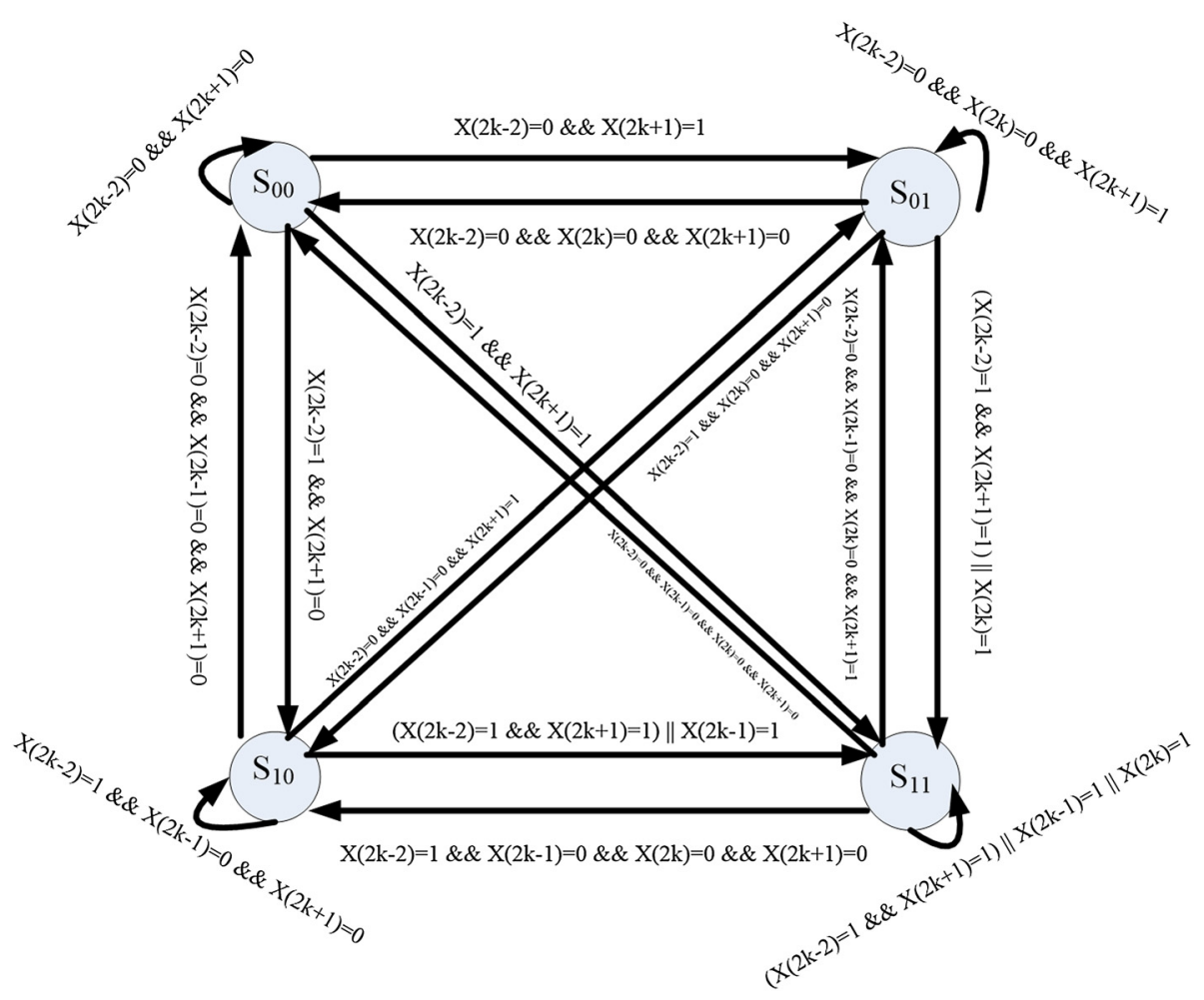

Figure 6 State transition diagram of Cluster_k of Figure 5.

The number of cells in each orthogonal group can be flexibly configured as depicted in Figure 3 . We set the orthogonal group to a range for each group that makes less impact on trains' speeds. The state of the group has a close relationship with the state of the cells. If all the cells in the group are in a passive state, that is to say, all BSs are in power-saving mode, the whole orthogonal group is in a passive state. Correspondingly, if even one cell in the orthogonal group is active, the state of the whole group is set to active. $g_{i}$ is the state of $i$ th orthogonal group, $h_{j}$ is the number of cells in this group. The state of orthogonal group is given by

$$
g_{i}=\left\{\begin{array}{l}
1 \text { (Active) }, c_{h_{1}} \| c_{h_{2}}|| \ldots|| c_{h_{j}}=1 \\
0 \text { (Passive) }, c_{h_{1}}|| c_{h_{2}}|| \ldots|| c_{h_{j}}=0
\end{array}\right.
$$

\subsubsection{State transition by dividing orthogonal groups into orthogonal clusters}

Let $m$ be the number of orthogonal groups. As a result, $2^{m}$ possible group states exist. The group state is transferred between different states depending on the present group state and the input event. Obviously, the state machine will become more complex with an increase in groups, because more groups mean more possible group states.

To solve the state transition problem in a more efficient manner, we use the divide and conquer method
[25] in this paper. In computer science, divide and conquer is an important algorithm design paradigm based on multi-branched recursion. The divide and conquer algorithm works by recursively breaking down a problem into two or more sub-problems of the same or related types, until these become simple enough to be solved directly. The solutions to the sub-problems are then combined to solve the original problem.

In Algorithm 1, the procedure from Step 3 to Step 14 shows how to construct orthogonal groups using input information from Steps 1 and 2. With the divide and conquer design paradigm, we divide the orthogonal groups

Table 2 Scenario parameters

\begin{tabular}{ll}
\hline Parameter & Value \\
\hline Number of BSs & 43 \\
BS core power & $60 \mathrm{~W}$ \\
Cell radius average & $3.9 \mathrm{~km}$ \\
Inter-cell distance standard deviation & $2.02 \mathrm{~km}$ \\
Power-saving strategies & $10 \%, 20 \%, 25 \%, 35 \%$ \\
Train scheduling time & $30 \mathrm{~min}$ \\
Train speed & $80 \mathrm{~km} / \mathrm{h}$ \\
Time interval for state & $10 \mathrm{~s}$ \\
$d_{\text {threshold }}$ & $3 \mathrm{~km}$ \\
\hline
\end{tabular}




\section{Algorithm 1 The outline of the proposed method}

1: Input: the number of cells $n$, the set of cells $C=\left\{c_{i}, \ldots, c_{n}\right\}$, the inter-cell distance $d_{i, i+1}(i=1, \ldots, n$ -1), the special cell indicator $\lambda_{i}$ (i.e., $c_{i}$ is a special cell if $\lambda_{i}=1$ ), predefined threshold inter-cell distance $d_{\text {threshold; }}$

2: Group (set $G=\left\{g_{1}=\varnothing, \ldots, g_{j}=\varnothing, \ldots\right\}$ ) formation based on the input information;

$3: j=1$;

4: FOR $i=1, \ldots, n$ DO

5: IF $\left(d_{i, i+1}<d_{\text {threshold }} \& \& d_{i-1, i} \geq d_{\text {threshold }}\right) \|\left(\lambda_{i}=1\right)$ THEN

6: $\quad c_{i}$ and $c_{i+1}$ are added into $g_{j}, g_{j}=g_{j} \cup\left\{c_{i}, c_{i+1}\right\}$;

7: $\quad$ ELSE IF $\left(d_{i, i+1} \geq d_{\text {threshold }} \& \& d_{i-1, i} \geq d_{\text {threshold }}\right) \|\left(\lambda_{i}=0\right)$ THEN

8: $\quad c_{i}$ is added into $g_{j}, g_{j}=g_{j} \cup\left\{c_{i}\right\}$;

9: ELSE IF $d_{i, i+1}<d_{\text {threshold }} \& \& d_{i-1, i}<d_{\text {threshold }}$ THEN

10: $\quad c_{i+1}$ is added into $g_{j-1}, g_{j-1}=g_{j-1} \cup\left\{c_{i+1}\right\}$;

11: END IF

12: $j=j+1$;

13: END FOR

14: The set of groups $G$ is formed and the element of $G$ is $m$ (i.e., $m$ groups);

15: Cluster formation based on divide and conquer method;

16: $\operatorname{FOR} j=1, \ldots, m$ DO

17: $g_{j}$ and $g_{j+1}$ are formed into cluster_ $(j+1) / 2$;

18: END FOR

19: $[\mathrm{m} / 2]$ clusters are obtained as a result;

20: State transition for BS power saving cluster by cluster;

21: WHILE (TRUE)

22: In each time interval $t$, wireless access request in each group is measured;

23: FOR $i=1, \ldots,[m / 2]$ DO

24: Cluster_i state transition is performed based on the measurement results;

25: END FOR

26: END WHILE 
into several orthogonal clusters which are outlined between Step 15 and Step 19, with each orthogonal cluster containing the same number of groups. As a result, performing the state transition of orthogonal groups in one orthogonal cluster becomes a sub-problem shown in the left steps of Algorithm 1. In this way, the solutions to sub-problems can first be obtained, and then combined, to achieve the final solution to the original problem.

\subsubsection{Detailed minimum orthogonal cluster solution}

To decrease the complexity to exploit the relationship between adjacent groups, a large state machine with $2^{m}$ group states is divided into $m / 2$ small state machines with four group states $(00,01,10,11)$ for each small state machine. We call the cluster with two groups the minimum orthogonal cluster, and the solution is to solve the subproblem with the group state transition in one cluster. Cluster_k in Figure 4 is selected to further illustrate how a small mealy-type state machine works to solve the subproblem. The details of Cluster_k are given in Figure 5.

Obviously, four possible states $(00,01,10,11)$ exist for Cluster_ $k$ as demonstrated in Table 1 . State transitions are conducted between the four states. In each time interval lasting $t$, the wireless access request of each orthogonal group is measured. For instance, we measure the wireless access request of each orthogonal group in the time interval $(t, 2 t)$ at time $2 t$. The results of measurement are the events or inputs to the related state machines, which will have an effect on the state transition following the measurement. The events or inputs can be described as follows:

$$
X(i)=\left\{\begin{array}{l}
1, \text { Wireles Access Request }_{\text {Group }_{i}}>0 \\
0, \text { Wireles Access Request }_{\text {Group }_{i}}>=0
\end{array}\right.
$$

Wireless_Access_Request_Group $i$ represents the wireless access request of the group $i$ in a certain time interval. $X(i)$ is an indicator of whether there is a wireless access request in group $i$. In addition, $X(i)$ plays the role of the input event to state machines in the proposed solution.

We propose the specific rules (i.e., the state transition function) for a state transition in each cluster. Figure 6 shows the state transition diagram for Cluster_ $k$ of Figure 5. A state transition is executed if events occur.

A state transition from $S_{00}$ to $S_{01}$ is used as an example to describe the procedures as shown in Algorithm 2. It is assumed that the present state of Cluster_k is $S_{00}$. The measurement module measures the wireless access request in each time interval, and measurement results are output to set the energy-saving mode. If no wireless access requests are detected in Group_2k-2 $(X(2 k-2)=0)$

\footnotetext{
Algorithm 2 State transition of Cluster_k from $S_{00}$ to $S_{01}$

1: Initial conditions: Cluster_k is in state $S_{00}$ (i.e., all the non-special BSs in Group_2k-1 and Group_ $2 k$ are in power saving model)

\section{2: WHILE(TRUE)}

3: In each time interval $t$, wireless access request in each group is measured;

4: Measurement results are used for judgment;

5: IF $X(2 K-1)=0 \& \& X(2 K+1)=1$ THEN

6: All the BSs belonging to Group_ $2 k$ should get out of power saving model;

7: $\quad$ State transition is executed and Cluster_k enters into state $\mathrm{S}_{01}$;

\section{8: ELSE THEN}

9: Other judgments are performed further for state transition;

10: END IF

\section{1: END WHILE}


and there are wireless access requests in Group_ $2 k+1$ $(X(2 k+1)=1)$, all the BSs in Group_2k are controlled to be out of the energy-saving mode. As a result, $S_{00}$ is transferred to $\mathrm{S}_{01}$.

The state transition for each cluster is performed simultaneously, and after the state transitions of all the clusters have been performed, the state of each orthogonal group is determined, which solves the original problem.

\section{Evaluation analysis}

In this section, we evaluate our energy-saving solution based on real GSM-R network data along a certain railway. Forty-three cells that cover the railway tracks between two BSs are selected to be optimized for BS energy consumption. More parameters can be found in Table 2 according to GSM-R network planning along the tracks. The algorithms and procedures for the simulations ${ }^{\mathrm{a}}$ are carried out using the Python language, which has functional and structured programming to handle railway simulations. First, we set up our simulation scenario for the power-saving statistics of each BS. In the time interval of state transition, trains can be scheduled to locations according to their speeds and scheduling times, and each BS calculates the power consumption using its cell state and power-saving strategy. Next, orthogonal groups are formed for these 43 cells. To our mind, it is important for the orthogonal group to make a low impact on the speed of the trains for the cells with a small cell radius. We use Figure 7 to show the inter-cell distances of the 43 cells. It shows some cells are close enough to assign them to one orthogonal group according to our solution. Here $d_{\text {threshold }}$ is highlighted for $3 \mathrm{~km}$, and for simplicity, we select one direction to create the orthogonal group which is carried by the BSC group in Figure 2 and is fixed for trains in both directions. As a result, cells with $\mathrm{BS}_{9}$ and $\mathrm{BS}_{10}$, and cells with $\mathrm{BS}_{24}$ and $\mathrm{BS}_{25}$ in Figure 7 are respectively assigned to one group. In the next three subsections, the BS energysaving ratio for the selected GSM-R network is investigated with the variation in BS power saving in the passive state, time interval for train scheduling, and train speed. In each of the three cases, the head and tail cells, which cover two stations, are always in the active state, that is to say, the two cells are not controlled for power saving.

\subsection{Impact of the RBS power-saving model}

Although it is not permissible for the system to entirely turn off some underused BSs in a low traffic period, because of the dedicated cellular railway network's safety requirements, there are also three power-saving strategies that can be implemented in a cellular railway network according to the BS architecture in Figure 1. A summary of the approximate energy-saving ratios is shown in Table 3. As previously mentioned, the BS should take action for power saving when a cell is in the passive state. To evaluate the impact of the amount of BS power saving on the energy-saving ratio for each BS within the selected GSM-R network, extensive simulations were conducted. We assume the train speed remained at $80 \mathrm{~km} / \mathrm{h}$, and the time interval for train scheduling was $30 \mathrm{~min}$. Figure 8 shows the simulation result. "Power saving 10\%" means the BS power can be reduced by $10 \%$ for power saving when the corresponding cell has been in the passive state. With the increase in the amount of BS power saving, higher BS energysaving ratios can be obtained. The BS energy-saving ratio cannot reach the amount of BS power saving because the cells along the railway are not always in a passive state. Besides, more energy saving can be achieved in the case of shorter inter-group distances.

\subsection{Impact of train schedule}

The time interval for scheduling trains has a considerable effect on the BS energy saving. If more trains pass through the railway, it will lead to longer times for BSs staying in their active state to serve these trains. Time intervals of $15,30,60$, and $120 \mathrm{~min}$, for scheduling trains, were chosen to evaluate our energy-saving solution. Moreover, during simulations, when the train speed was $80 \mathrm{~km} / \mathrm{h}$, the BS power reduced by $35 \%$ when the corresponding cell was in the passive state. As demonstrated in Figure 9, a longer time interval for scheduling trains results in a higher BS energy-saving ratio, because more BSs are stayed in the passive state. We also noticed that when the time interval for scheduling trains was short enough, some BSs belonging to groups with long inter-group distances did not enter into the powersaving model. A shorter time interval for scheduling trains means more trains running on the railway, and a longer inter-group distance means that trains need more time to pass through these groups, both of which lead to the phenomenon.

\begin{tabular}{llc}
$\begin{array}{l}\text { Table } 3 \text { Energy saving ratio with power-saving } \\
\text { strategies }\end{array}$ & $\begin{array}{l}\text { Energy saving } \\
\text { ratio (\%) }\end{array}$ \\
\hline Power-saving strategy & Feature & 20 \\
\hline Activation on radio unit & $\begin{array}{l}\text { BTS power saving/time slot } \\
\text { power saving [24,26] }\end{array}$ & 10 \\
$\begin{array}{l}\text { Activation on cooling } \\
\text { system }\end{array}$ & Passive cooling [27] \\
$\begin{array}{llc}\text { Activation on radio unit } \\
\text { and DC power unit }\end{array}$ & $\begin{array}{l}\text { DC power saving related } \\
\text { on radio unit }\end{array}$ \\
$\begin{array}{lll}\text { Activation on all three } \\
\text { parts }\end{array}$ & All & 25 \\
\hline
\end{tabular}




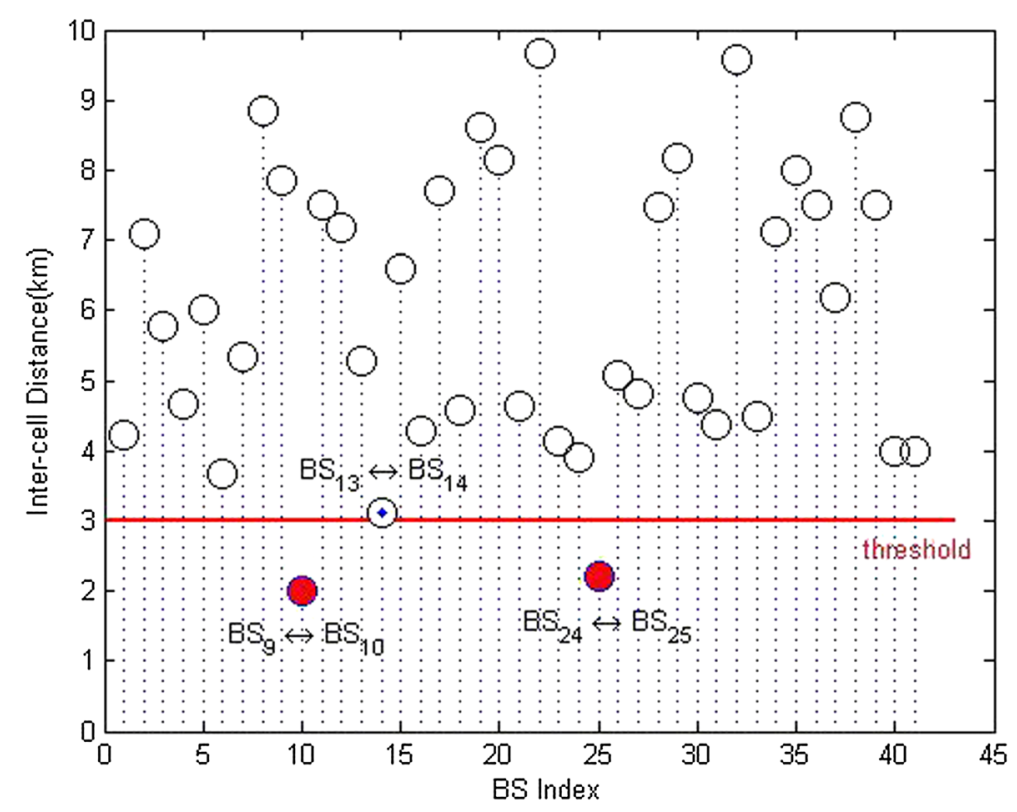

Figure 7 Inter-cell distances of the 43 cells.

\subsection{Impact of train speed}

The speed of trains was also investigated to evaluate the proposed solution for BS energy saving. The variation in train speed had an effect on the time taken by a train to pass through a group. In the simulations, the time interval for scheduling trains was $30 \mathrm{~min}$, and the BS power reduced by $35 \%$ for power saving when the corresponding cell was in the passive state. Figure 10 shows that a higher train speed brings about a higher BS energysaving ratio, because trains passed through a group in a shorter period of time. Thus, BSs could remain in the power-saving mode longer. In addition, Figure $10 \mathrm{dem}$ onstrates that the BS energy-saving ratio was much closer when the speed of the trains was great enough, because trains passed through a group in a very short time when at high speed.

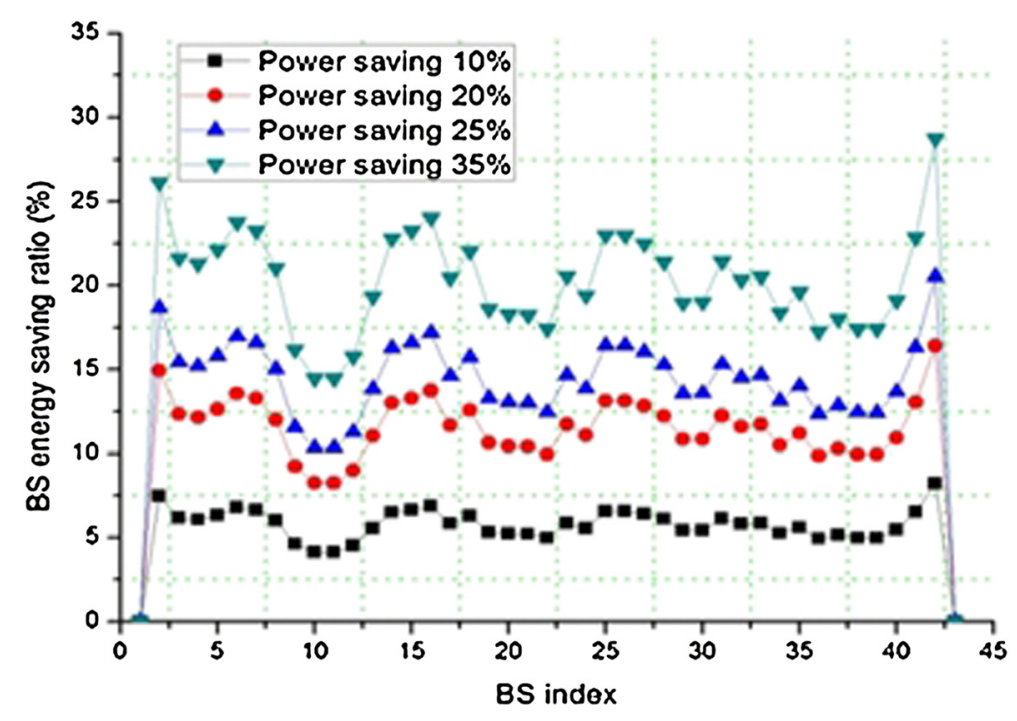

Figure 8 BS energy-saving ratio with variation of BS power saving in the passive state. 


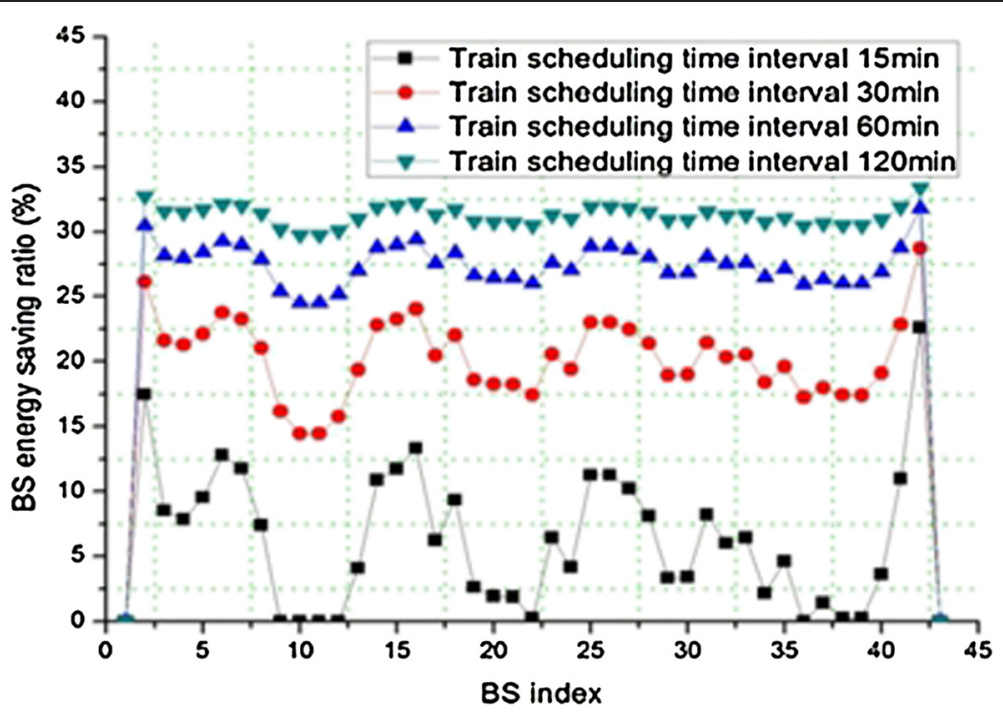

Figure 9 BS energy-saving ratio with variation of time interval for scheduling trains.

\section{Conclusions}

In this study, we focused on the issue of a dynamic energy-saving strategy in a cellular railway network. According to the practical analysis, we concluded that energy saving was necessary for cells that did not serve as a vehicle station. Taking into account the difficulty of collecting the train schedules in advance, we proposed a train detection strategy based on the handover process. Furthermore, a mealy-type finite state machine-based solution for BS energy saving along railway tracks was proposed, which flexibly and effectively transferred the cell's state and can be applied to power-saving models. Finally, we built our simulating environment based on a real cellular railway network, and the numerical results showed that energy saving can be achieved using different power-saving models, train scheduling, and speed, providing a promising solution for dynamically reducing energy consumption for BSs along railway tracks. Future study will involve more cellular networks which can provide communication services for both railway and the habitants along the tracks.

\section{Endnotes}

${ }^{a}$ In the evaluation part, we only implemented the power-saving strategy using the Python language on the top layer. We did not touch any implementation of

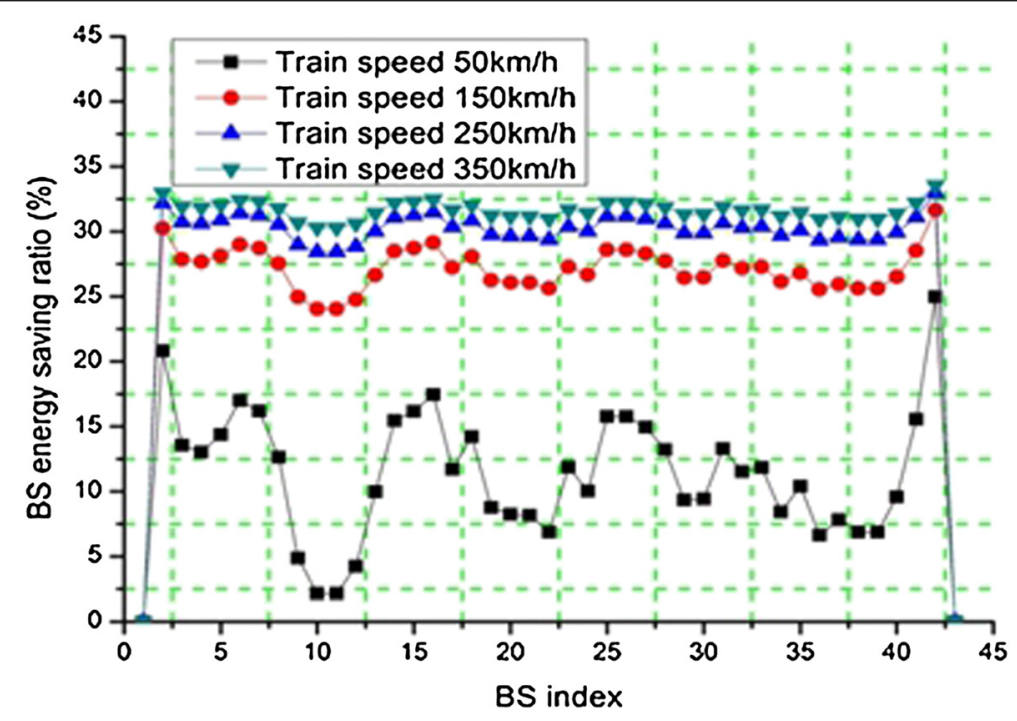

Figure 10 BS energy-saving ratio with variation in train speed. 
physical (PHY) layer. We believe that this is sufficient to prove the effectiveness of our strategy because we use the HANDOVER message to detect the train arrival in the detection part of our power-saving strategy. Handover is the normal procedure when a vehicle station enters from one cell to another according to the 3GPP Standard. The HANDOVER message is the mandatory message for the handover procedure. We propose the power-saving strategy on the basis of the handover procedure. Therefore, without any modification for PHY layer in the GSM-R railway network, our strategy can be directly carried out on the BSC part as the operators' view. However, for the data and topology (shown in Figure 2), we used the evaluations that are all based on the real scenario. As the data list is too long to show in this article, we directly evoke the database in our evaluation. For the detailed raw data of the topology, real signaling capture and simulation results, please access http://pan. baidu.com/s/1ntoO2gx or http://eaie.bjtu.edu.cn/rawdata_ Green_CELL.zip to download, if there are any further questions, please contact ejiying@126.com.

For the same reason, it is also unnecessary to evaluate the robustness of our evaluation. The power-saving strategy will be activated only by the parameter of detecting the HANDOVER message. As mentioned above, the handover procedure is robust and reliable in the GSM-R network because of its high security requirements. Therefore, we can use the message to ensure our solution's robustness.

\section{Competing interests}

The authors declare that they have no competing interests.

\begin{abstract}
Acknowledgements
The authors express their thanks for the support from the National Science Foundation of China under Grant 61222105, the Beijing Municipal Natural Science Foundation under Grant 4112048, the Program for New Century Excellent Talents in Universities of China under Grant NCET-09-0206, the NSFC under Grant 60830001, and the Key Project of State Key Lab under Grant no. RCS2011ZZ008. Portions of this article were previously presented at IEEE VTC2014 Spring, titled, Dynamic green communication strategy for railway cellular network, April 2014.
\end{abstract}

\section{Author details \\ ${ }^{1}$ School of Electronic and Information Engineering, Beijing Jiaotong University, No. 3 Shangyuancun, Beijing 100044, China. ${ }^{2}$ School of Computer and Information Technology, Beijing Jiaotong University, No. 3 Shangyuancun, Beijing 100044, China. ${ }^{3}$ China Science and Technology Exchange Center, No. 54 Sanlihe Road, Beijing 100045, China.}

Received: 2 April 2014 Accepted: 2 March 2015

Published online: 24 March 2015

\section{References}

1. McLaughlin S (2008) Green radio: the key issues programme objectives and overview. Wireless World Research Forum, Stockholm, Sweden, October 13-15, 2008. Available on http://wwrf.ch/files/wwrf/content/files/publications/libraries/ Library21.pdf

2. J Hansen, M Sato, P Kharecha, G Russell, DW Lea, M Siddal, Climate change and trace gases. Philos Trans RSoc 365(1865), 1925-1954 (2007)
3. A Fehske, G Fettweis, J Malmodin, G Biczok, The global footprint of mobile communications: the ecological and economic perspective. IEEE Commun Magazine 49(8), 55-62 (2011)

4. Z Hasan, H Boostanimehr, VK Bhargava, Green cellular networks: a survey, some research issues and challenges. Commun Surveys Tutorials, IEEE 13(4), 524-540 (2011)

5. D Antonio De, S Emilio Calvanese, C Antonio, Enabling green cellular networks: a survey and outlook. Comput Commun 37, 5-24 (2014). ISSN 0140-3664

6. SA Waje, SB Rahane, A survey of Green wireless communications, international journal of electronics communication and instrumentation engineering research and development (IJECIERD), ISSN 2249-684X, Vol.3, Issue2, 25-36, June, 2013

7. X Wang, AV Vasilakos, M Chen, Y Liu, TT Kwon, Taekyoung. A survey of green mobile networks: opportunities and challenges. Networks Appl 17, 4-20 (2012)

8. S Roy, Energy Logic for Telecommunications, Emerson Network Power Energy Systems, North America, Inc, September, 2008. http://www. emersonnetworkpower.com/documents/en-us/brands/energysystems/ documents/netxtend\%20pages/energy\%20logic\%20for\%20telecom\% 20white\%20paper.pdf (webpages accessed on February 8, 2014

9. White Paper Ericsson (2007). Sustainable energy use in mobile communications white paper. EAB-07:021801, Ericsson AB, Stockholm, Sweden, 2007

10. A Ani Vincent, O Ani Emmanuel, Assessment of carbon credits for power generation systems at GSM base station site. Int J Renew Energy Res 3(4), 763-768 (2013)

11. K Son, H Kim, Y Yi, B Krishnamachari, Toward energy-efficient operation of base stations in cellular wireless networks, Green communications: theoretical fundamentals, algorithms, and applications (CRC Press, Taylor \& Francis, Boca Raton, FL, USA, 2012)

12. E Oh, K Son, B Krishnamachari, Dynamic base station switching-on/off strategies for green cellular networks. IEEE Trans Wirel Commun 12(5), 2126-2136 (2013)

13. GSM-R. http://en.wikipedia.org/wiki/GSM-R. (webpages accessed on August 31, 2013.)

14. R Md Moklesur, Overview of energy saving aspects in $2 G$ and $3 G$ mobile communication networks, Master's thesis (University of Gavle, Sweden, 2009)

15. Ericsson Psi. http://www.ericsson.com/ourportfolio/telecom-operators/psicoverage

16. Flexenclosure eSite. http://www.flexenclosure.com/esite/

17. L-CWang, S Rangapillai, A survey on green $5 \mathrm{G}$ cellular networks, in Proceedings of 2012 International Conference on Signal Processing and Communications (SPCOM), Bangalore, India, pp.1-5, 22-25 July 2012

18. X Zhang, S Zhuowen, Z Yan, W Wang, Energy-efficiency study for two-tier heterogeneous networks (HetNet) under coverage performance constraints. Mobile Networks Appl 18(4), 567-577 (2013)

19. D Calin, H Claussen, H Uzunalioglu, On femto deployment architectures and macrocell offloading benefits in joint macro-femto deployments. IEEE Commun Mag 48(1), 26-32 (2010)

20. Y Xiao, van der Schaar M, Energy-efficient nonstationary power control in cognitive radio networks, in Proceedings of IEEE GLOBECOM, IEEE Communications Society, Atlanta, GA, USA, pp. 3029-3034, December 9-13, 2013

21. MA Marsan, L Chiaraviglio, D Ciullo, M Meo, Optimal energy savings in cellular access networks, in Proceedings of 1st International Workshop on Green Communications (IEEE GreenComm 2009), pp. 1-5, IEEE, Dresden, Germany, June 14-18, 2009

22. C Peng, S Lee, $S$ Lu, H Luo, H Li, Traffic-driven power saving in operational $3 G$ cellular networks, in Proceedings of the 17th Annual International Conference on Mobile Computing and Networking, (MOBICOM 2011), pp. 1-12, Las Vegas, Nevada, USA, September 19-23, 2011

23. L Zhang, J Zhu, Z Tian, Research on verification method of mutual authentication in GSM/UMTS inter-system based on finite state machine, in Proceedings of International Conference on IMCCC, 2011, Instrumentation, Measurement, Computer, Communication and Control, (IMCCC 2011), pp. 541-544, Beijing, China, October 21-23, 2011

24. C Hong, A study on intelligent electricity saving technology in GSM system. ZTE Technologies 8, 24-25 (2008)

25. Divide and conquer algorithms. Available on https:/en.wikipedia.org/wiki/ Divide_and_conquer_algorithm 
26. L Qianhao, Z Jie, Application analysis of light and energy saving GSM BTS. ZTE Technologies 8, 26-27 (2008)

27. C Lubritto. Telecommunication power system: energy saving, renewable sources and environmental monitoring, Trends in telecommunications technologies, Trends in Telecommunications Technologies, Christos J Bouras (Ed.), Intech, Rijeka, Croatia, March 1, 2010 ISBN: 978-953-307-072-8, 2010. Available from: http://www.intechopen.com/books/trends-intelecommunications-technologies/telecommunication-power-systemenergy-saving-renewable-sources-and-environmental-monitoring

Submit your manuscript to a SpringerOpen ${ }^{\odot}$ journal and benefit from:

- Convenient online submission

- Rigorous peer review

- Immediate publication on acceptance

- Open access: articles freely available online

- High visibility within the field

- Retaining the copyright to your article

Submit your next manuscript at $>$ springeropen.com 